Revista Hechos y Proyecciones del Lenguaje. No. 9. Universidad de Nariño -UNED. (ISSN: 0121-3350). (pp. 8090). 1995.

APA Citation Style: Benavides B., Jorge E. (1995). El Auge de los Computadores: el Futuro del Aprendizaje de los Idiomas. Revista Hechos y Proyecciones del Lenguaje (9), 80-90.

\title{
El Auge de los Computadores: el Futuro del Aprendizaje de los Idiomas
}

\author{
Jorge E. Benavides B. (joelbebu@gmail.com) \\ Departamento de Lingüística e Idiomas \\ Universidad de Nariño, San Juan de Pasto, Colombia
}

\section{Introducción}

El nuevo campo del aprendizaje de idiomas por medio del computador ha despertado muchas expectativas a nivel nacional y esto se está convirtiendo cada vez más en una posibilidad real para estudiantes y profesores quienes tienen interés en aplicar los avances de la tecnología en el campo de la informática educativa.

Sin embargo, existen muchos escépticos, sobre todo, profesores y pedagogos (Benavides, 1989) quienes es alguna forma -y con cierta razón- han venido cuestionando las actuales Prácticas pedagógicas y educativas con los computadores y los programas utilizados con estudiantes.

Antes de echar un vistazo a algunas de las posibilidades de los computadores en el campo del aprendizaje de los idiomas es necesario familiarizarnos con dos términos que ya han hecho carrera lingüística en nuestro medio. Términos que se han introducido del inglés y por no tener un equivalente en español se utilizan directamente con algunas variaciones de pronunciación más no de forma.

Hardware, se refiere a la parte física, visible que compone un computador. Esta parte está compuesta por todos los elementos físicos, mecánicos y electrónicos, como el procesador, la memoria, circuitos, etc., que intervienen en el procesamiento de la información. De la misma manera hacen parte del hardware otros componentes del computador que van conectados a éste como son el monitor, la impresora, el teclado (dispositivos de input y output). 
Revista Hechos y Proyecciones del Lenguaje. No. 9. Universidad de Nariño -UNED. (ISSN: 0121-3350). (pp. 8090). 1995.

Software se refiere a todos aquellos programas tanto de sistema como de aplicación que una vez en la memoria del computador son los encargados de dirigir las operaciones de la máquina.

Existen en el momento diferentes clases de aspectos relacionados con el desarrollo del aprendizaje de idiomas mediante el computador (de aquí en adelante AIMC).

\section{APRENDIZAJE DE IDIOMAS MEDIANTE EL COMPUTADOR (AIMC)}

La idea de utilizar computadores en las clases de idiomas ha despertado una gran cantidad de reacciones y expectativas, en nuestro medio con el capítulo de (Computer-Assisted Language Learning dentro de la Asociación Colombiana de Profesores de Inglés, ASOCOPI). Esto el resultado de la introducción de la tecnología educativa a través del computador lo que hace posible la implementación y desarrollo de actividades completamente nuevas - de las consideradas tradicionales- para el estudiante de idiomas lo mismo que para el profesor. Estas actividades han sido implementadas a partir de aplicaciones y trabajos sencillos como los de los procesadores de texto, las hojas electrónicas, las bases de datos, los juegos didácticos, hasta los programas más nuevos y sofisticados como los que están disponibles (a pesar de no estar aun pedagógicamente implementados) con los últimos desarrollos en Hardware y Software: multimedios, inteligencia artificial, manejo de sistemas expertos, etc.

\section{ETAPAS EN EL DESARROLLO DE (AIMC)}

El aprendizaje de idiomas mediante el computador se entiende hoy como el uso de los computadores como mecanismo para suplementar el aprendizaje de los idiomas. Desafortunadamente, esta utilización se encuentra en una etapa incipiente de introducción (nuestra actual etapa) donde los resultados en términos de aprendizaje sólo provienen de las expectativas aisladas realizadas por profesores entusiastas mediante reportes sucintos de aprendizaje en estudiantes (Benavides, 1993).

Una segunda etapa en el aprendizaje de idiomas mediante el computador tendría que relacionarse con la familiarización de los usuarios (administradores de la educación, profesores 
Revista Hechos y Proyecciones del Lenguaje. No. 9. Universidad de Nariño -UNED. (ISSN: 0121-3350). (pp. 8090). 1995.

y estudiantes) con las nuevas técnicas basadas en el uso adecuado de los computadores donde se pretende realizar aplicación, implementación e investigación acerca de cómo se aprende mediante el uso de esta tecnología.

La investigación sistemática de los aspectos de aprendizaje concernientes a la lengua y sus resultados serían la base para la consolidación de la tercera etapa en este proceso, donde los resultados de la investigación sistemática sobre aplicación, implementación, y aprendizaje serian la base de una filosofía pedagógica del uso de los computadores. La integración de estos resultados a los currículos de la lengua tanto nativa como extranjera seria el producto de un trabajo consistente de investigación, aplicación e implementación de los computadores en esta área específica. Se alcanzaría así el ideal de concebir y realizar la docencia con base en la investigación sistemática.

\section{QUE NOS PUEDE OFRECER DE NUEVO EL (AIMC)?}

Es necesario que nos cuestionemos, y usualmente la gente lo hace, acerca de algunos aspectos importantes para luego entrar a dar una respuesta. Lo que la gente se pregunta acerca del uso de los computadores en educación y más concretamente en el campo del aprendizaje de idiomas tienen que ver con las siguientes preguntas:

1. ¿Qué contextos pueden crearse mediante el uso del computador?

2. ¿Cuál es la relación entre el estudiante- computador y profesor-computador?

3. ¿Qué clase de habilidades del lenguaje pueden estimularse y mejorarse con el uso del computador?

4. ¿A qué punto puede servir el computador para la pedagogía comunicativa?

5. ¿Cuál es la relación de lo que hace el computador con el punto de vista entre aprendizaje y adquisición?

6. ¿Puede decirse que el uso del computador en este campo constituye una actividad humanística? 
Revista Hechos y Proyecciones del Lenguaje. No. 9. Universidad de Nariño -UNED. (ISSN: 0121-3350). (pp. 8090). 1995.

Mi intención no es la de responder la totalidad de las preguntas anteriores, para ello necesitaría de algo más de un libro. Sin embargo podría iniciar este aspecto a modo de información con una de las preguntas claves, es decir, ¿qué clase de habilidades y en qué medida pueden generar o impulsar el uso de los computadores en el campo del aprendizaje de los idiomas?

\section{CLASES DE ACTIVIDADES EN (AIMC)}

En el momento, una actividad poco probable con el computador sería una de tipo conversacional en la que el estudiante interactúe con el computador como si éste fuera no sólo un 'entendedor' sino un practicante del acto de habla. Sin embargo, existe una amplia gama de actividades interactivas para las cuales el computador puede ser una buena alternativa de desarrollo de habilidades lingüísticas y de conocimiento en general (Philips, 1985).

Si se considera y se trabaja sobre el supuesto de que diferentes clases de actividades generan diferentes clases de aprendizajes y de conocimientos, éstas pueden enfocarse a puntos o aspectos bien discretos en algunas de las habilidades del lenguaje. Esto permitiría en teoría desarrollar diferentes estrategias de aprendizaje por parte de los estudiantes.

En términos generales cada tipo de actividades en (AIMC) le brinda la posibilidad al estudiante de desarrollar una tarea, cuya culminación implica el uso de habilidades lingüísticas y conocimientos lingüísticos. Al finalizar la tarea o actividad se habría alcanzado un objetivo el cual se denominaría el objetivo lingüístico principal.

Adentrándonos un poco más en el tipo de actividad que presentaría el computador y que desarrollaría el estudiante con un objetivo lingüístico, se puede manifestar que el computador es una maquina o herramienta muy versátil que puede generar una amplia gama de actividades para desarrollar un objetivo de aprendizaje. Este puede incluirse en juegos, cuestionarios, construcción o reconstrucción de texto, simulaciones, exploraciones, solución de problemas, etc. Es importante añadir a este respecto que las anteriores actividades no se podrían realizar mediante cualquier otro medio, mucho menos con el texto. 
Revista Hechos y Proyecciones del Lenguaje. No. 9. Universidad de Nariño -UNED. (ISSN: 0121-3350). (pp. 8090). 1995.

Los programas que presentan un formato de juego pueden alcanzar un objetivo lingüístico por medio de una estrategia a seguir por el estudiante en un contexto donde se enfrenta al computador, o mediante el computador a otro estudiante. De todas maneras el computador hace posible que el objetivo se presente en el transcurso del juego y se alcance si el estudiante termina la actividad.

Un ejemplo de la anterior actividad puede encontrarse en el programa WORDSNAP, en el que el estudiante compite con el computador tratando de suministrar palabras que se necesitan en determinado contexto. En este juego se pueden encontrar diferentes niveles de dificulta. Por ejemplo, desde suministrar la palabra correcta sin tener ninguna pista hasta poder elegir de una serie de posibles respuestas.

Los crucigramas y rompecabezas son programas que generan otro tipo de actividad que pretende ejercitar al estudiante a nivel de vocabulario y gramática sobre la forma más adecuada de formar palabras, frases, oraciones, en diferente orden o trabajando con gráficos en un contexto más realista (Bloom, 1970). Estos programas requieren de un conocimiento de la morfología del idioma en cuestión. Estos programas se conocen también como programas de construcción de texto, y pueden también trabajar con ejercicios de selección múltiple.

Otro tipo de programas se encuentran en los de reconstrucción de texto como STORYBOARD o QUARTEXT. Estos programas generan actividades para analizar textos o pasajes ya existentes en la memoria del computador. Aquí el esfuerzo del estudiante se centra en el hecho de tratar de recuperar un texto que ha sido manipulado por el computador de acuerdo a diferentes niveles de dificultad. El estudiante puede escoger de entre diversas posibilidades que le ofrece el computador la forma de reconstrucción, inclusive una de tipo competitivo con el computador en el que el objetivo es tratar de completar un texto acertando la palabra adecuada. Para esto el computador puede brindar ayuda cada vez que el estudiante se lo pida. Por ejemplo, en una palabra difícil, le puede dar la primera, segunda, tercera, etc., letra para poder completar la tarea. Claro está que a mayor número de pistas y ayuda, menor será el puntaje alcanzado al finalizar la actividad. 
Revista Hechos y Proyecciones del Lenguaje. No. 9. Universidad de Nariño -UNED. (ISSN: 0121-3350). (pp. 8090). 1995.

Uno de los tipos de programas que más prometen en este campo son los diseñados mediante lenguaje de cuartas generaciones o inteligencia artificial en lo que el computador se programa con un conjunto de reglas para aspectos específicos de la lengua a aprender.

\section{CARACTERISTICAS TECNICO-PEDAGOGICAS DE LOS MATERIALES (AIMC).}

Desafortunadamente, gran cantidad de programas utilizados en el aprendizaje de los idiomas parecen no tener consistencia o relación con determinada corriente pedagógica y sobre esto existe muchos críticos que dudan de la confiabilidad de las ventajas asignadas a las actividades en (AIMC). Sin embargo, está bien claro que el esfuerzo de los diseñadores y productores de materiales en este campo por salirse de la línea tradicional de instrucciones como la que se realiza en el salón de clases, puede dar lugar a inconsistencias. Sin embargo la mayoría de programas (AIMC) puede ayudar a los estudiantes a reorientar sus estilos de aprendizaje y a enriquecer sus nuevas estrategias de aprendizaje. Con ellos se enfatiza la necesidad de la práctica, a diferencia de la enseñanza e instrucción explícitas.

Las siguientes son algunas de las características más comunes de los programas de computador utilizados en (AIMC) y que se requieren para su uso consistente en el manejo de los mismos y para que la evaluación de resultados se haga en forma más confiable.

1. Producción automática de ejercicios

2. Suministro de corrección y retroalimentación

3. Desarrollo interactivo de las sesiones entre el usuario y el computador

4. Suministro de resultados y análisis de los mismos después de una determinada sesión

5. Utilización amistosa (user-friendly) del programa (eliminando las ambigüedades en lo posible)

6. Suministro de opciones diversas dentro del programa (selección de ejercicios varios, acceso de las respuestas, acceso a pistas, opción para parar, continuar, o terminar)

7. Presentación visual atractiva

8. Disponibilidad de niveles de dificultad 
Revista Hechos y Proyecciones del Lenguaje. No. 9. Universidad de Nariño -UNED. (ISSN: 0121-3350). (pp. 8090). 1995.

\section{Dirección personalizada}

Programas con inclusión de aspectos tecnológicos más avanzados y que se requieren en determinadas instituciones hacen uso de voz sintética para proveer corrección o input auditivo como comprensión de escucha en el aprendizaje (Ariew \& Dunkel, 1989).

No está muy lejana la adición de aspectos tales como el reconocimiento de la voz humana por parte del computador para lograr una interacción más realista entre la máquina y el usuario diferente a la meramente visual o escrita como la que utilizamos hoy en día.

\section{EL PAPEL DEL PROFESOR}

Generalmente no se piensa que tanto profesor como computador pueden hacer un trabajo en conjunto. Mientras que al profesor con su texto y con su grabadora y cassettes se los mira como aliados íntimos, la relación profesor computador se mira cautelosamente como de rivalidad. De esta manera se obscurece el hecho de que ambos pueden funcionar juntos. Sin embargo, y con base en experiencias recientes, en las que el profesor en realidad puede recibir un desarrollo más significativo que en otras áreas, se prevé que el profesor tendrá al computador no sólo como su aliado si no como su asesor. Consecuentemente, el profesor que trabaja en (AIMC) tendrá mejores prospectos de trabajo en el futuro (Benavides, 1993).

De todas maneras, el propósito de la utilización de los computadores en el campo de aprendizaje de los idiomas no es el desplazamiento de profesor a un plano secundario. Por el contrario se necesitarán más profesores y monitores encargados del aprendizaje de los idiomas. Es importante aclarar que mucha gente tiene miedo de la apertura hacia los computadores aduciendo que la máquina va a desplazar al hombre. Probablemente haya algo de cierto en ello, pero esto sólo se relaciona en forma relativa a la producción de elementos en serie. En educación no se puede hablar de que el computador va a remplazar al profesor ya que, con la introducción del computador en el aprendizaje de los idiomas se podría pensar que en realidad puede realizarse la idea de que el profesor sea un guía menos explícito para el estudiante en su aprendizaje. 
Revista Hechos y Proyecciones del Lenguaje. No. 9. Universidad de Nariño -UNED. (ISSN: 0121-3350). (pp. 8090). 1995.

El papel del profesor se puede plantear desde el punto de vista de aceptación de los computadores como un medio de aprendizaje suplementario. Una vez alcanzado un grado de concientización en este punto, nos preguntaríamos: ¿Qué pueden hacer los profesores para que los computadores se vuelvan instrumentos o herramientas de aprendizaje? Para alcanzar esta meta podemos sentar como prerrequisito, que el profesor interesado en los computadores en educación asuma los siguientes roles: un usuario del curso educacional (programas), un adaptador de materiales suplementarios; o un creador de cursos educacionales (courseware).

\subsection{Usuarios de que paquetes comerciales de aplicación.}

En este caso la institución interesada adquiere una serie de programas que son distribuidos comercialmente y los asigna a los estudiantes para que los utilicen. De antemano se supone que estos materiales han sido objeto de una cuidadosa evaluación de acuerdo con las necesidades del estudiante y los objetivos institucionales. Sin embargo, muchas veces se presentan casos en los que tanto profesores como estudiantes tienen que utilizar cualquier paquete de programas que la institución haya adquirido y tratar de hacer uso de ellos lo mejor posible.

\subsection{Adaptadores de materiales suplementarios.}

Los profesores pueden adaptar o modificar materiales ya existentes (programas) para complementar los materiales que ya poseen. En este caso, deben tener un entrenamiento práctico en el análisis de programas, evaluación y juicio crítico para determinar qué clase de adaptación o modificación necesitan los programas en cuestión. En este momento es conveniente insistir que la mayoría de paquetes de software educacional (conjunto de programas) son factibles de ser modificados e incluso de ser mejorados. Claro está que esto sólo se puede hacer si tanto estudiantes como profesores se adentran en la revisión crítica de software educacional o en el desarrollo del mismo. 
Revista Hechos y Proyecciones del Lenguaje. No. 9. Universidad de Nariño -UNED. (ISSN: 0121-3350). (pp. 8090). 1995.

\subsection{Creadores de materiales completos.}

Los profesores, pueden en otra situación, crear o desarrollar un conjunto completo de programas acompañados de sus respectivos manuales, e instrucciones. Esto, por supuesto requiere del conocimiento de un diseño instruccional, y la forma cómo todos los temas y tópicos van a ser presentados. Además se requiere que el profesor maneje al menos un lenguaje de programación y que conozca los procedimientos básicos del computador para que sea acorde con el área de aplicación, en este caso, la enseñanza-aprendizaje de los idiomas. Analógicamente con los escritores de textos, el profesor que se adentre a la creación y desarrollo de materiales informáticos debe tener un conocimiento necesario de la metodología y los contenidos en la enseñanza de un idioma.

La disponibilidad de computadores (hardware) tiene una gran ventaja sobre la disponibilidad de materiales o cursos educacionales (software). Sin embargo los profesores pueden hacer algo más que esperar a que los materiales sean desarrollados o que estén a la mano para suplir sus necesidades. Esto nunca sucederá, y los profesores tendrán que adentrarse al desarrollo de cursos y materiales educativos y adquirir la pericia en la evaluación de los cursos mismos o de otros a nivel comercial. Sin embargo, no sería realista esperar que cada profesor se interese o tenga que desarrollar esta clase de materiales. Sería tan absurdo como pensar que todos los profesores están interesados y/o tengan que escribir textos. No obstante, es una expectativa realista tratar de capacitar profesores para que desarrollen materiales suplementarios y/o que se asesoren del potencial que puede desarrollar un computador y un programa de computador. Además, esto les permitirá generar una crítica evaluativa de los materiales que se pueden utilizar en el aprendizaje de los idiomas. Esto parece más realista como el hecho de que los profesores sean capaces de hacer carteleras, sono-visos, cuadros, y conferencias para facilitar el aprendizaje en el aula de clase y los contenidos de un texto guía.

Sobra decir que los papeles arriba mencionados requieren que el usuario, (el profesor, en este caso), posea los conocimientos básicos de cómo operar los equipos. Por consiguiente, el primer paso para este conocimiento es saber qué clase de equipos se utilizan en el laboratorio de 
Revista Hechos y Proyecciones del Lenguaje. No. 9. Universidad de Nariño -UNED. (ISSN: 0121-3350). (pp. 8090). 1995.

computadores, sus características educacionales y su aplicación. Luego vendrá más fácil la parte del uso pedagógico del material.

Lo más crítico en el momento es la carencia de un programa unificado y de un trabajo conjunto para el inicio de un programa basado en (AIMC), por ejemplo, en donde después de la adquisición de los equipos y materiales, se diseñaría la forma de aplicación de esos materiales para que suplan diferentes necesidades de aprendizaje y en diferentes niveles.

\section{Conclusión}

Debemos tener en cuenta que en nuestro medio estamos experimentando una etapa de introducción al uso de los computadores y de pre-introducción de los mismos en el campo educativo. Esta etapa debe acogerse en forma decidida para conocer las ventajas reales de los materiales computacionales en la educación. A esta etapa seguiría, como se anotaba anteriormente, una de familiarización con los computadores en la que tanto educandos como educadores conoceríamos el potencial de aplicación de los computadores que nos ha dado su utilización inicial. Finalmente, nos encontraríamos con una etapa de integración del usuario con el computador y sus aplicaciones en el currículo con la consiguiente concientización de la necesidad del computador en los aspectos de aprendizaje de un idioma. Su puesto se aseguraría como imprescindible en la educación del futuro.

La introducción de una innovación está sujeta a experiencias iniciales que redundan en una adecuación de la innovación misma. El profesor en esta forma se ira convirtiendo en una especie de analista de software (como lo haría con un texto) que evalúa sus materiales para el mejor uso y provecho de los estudiantes, en este campo, además de su apoyo pedagógico, en la investigación y en la experimentación sistemáticas. 
Revista Hechos y Proyecciones del Lenguaje. No. 9. Universidad de Nariño -UNED. (ISSN: 0121-3350). (pp. 8090). 1995.

\section{REFERENCIAS}

Ariew, Robert. y P. Dunkel. (1989). ' $\underline{\text { A prototype for a computer-based listening comprehension }}$ proficiency test'. [ERIC database].

Bloom, Lois. (1970). 'Language Development: From and Function Emerging Grammars'. Cambridge, MA: MIT Press.

Benavides B., Jorge E. (1989). 'Iniciación a la Instrucción Complementaria del Ingles Mediante Computadores y Programación'. Revista de Investigaciones, Universidad de Nariño. 3(5), pp. 170-193,

Benavides B. Jorge E. (1993). 'Informática Educativa para el Aprendizaje de los idiomas'. Revista de investigaciones, Universidad de Nariño. 6(1), pp. 196-206,

Philips, M. (1985). 'Logical Possibilities and classroom scenarios for the development of CALL', in Brumfit, M. Philips and P. Skehan (eds). Computers in English Language Teaching. Oxford: Pergamon Press, pp. 25-46. 